\title{
Leprosy elimination campaign in Qazvin province, Islamic Republic of Iran (2006-07)
}

R. Qasemi-Barqi, ${ }^{1}$ B. Bijani ${ }^{1}$ and A.A. Pahlevan ${ }^{2}$

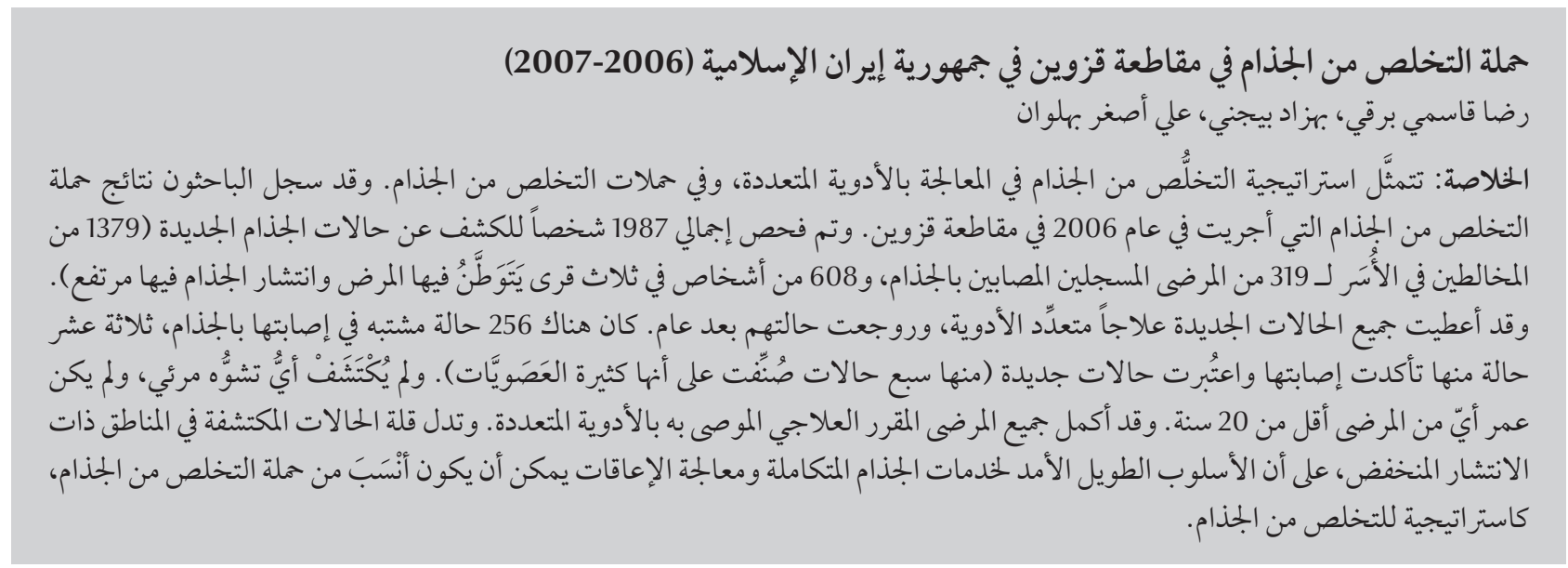

ABSTRACT Multi-drug therapy (MDT) and Leprosy Elimination Campaigns (LEC) are the major strategies for eliminating leprosy. We report the results of a LEC conducted in 2006 in Qazvin. A total of 1987 individuals (1379 household contacts of 319 registered leprosy patients and 608 people from 3 endemic villages with a high prevalence of leprosy) were examined for detection of new cases of leprosy. All new cases were given MDT and were reviewed after a year. There were 256 suspected cases of leprosy, 13 of whom were confirmed as new cases ( 7 were classified as multibacillary leprosy). None had visible deformity nor was $<20$ years old. All patients completed the recommended MDT course. The few cases detected suggest that in low prevalence areas, a longterm approach of integrated leprosy services and disability management may be more appropriate than LEC as a leprosy elimination strategy.

\section{Campagne pour l'élimination de la lèpre dans la province de Qazvin de 2006 à 2007 (République islamique} d'Iran)

RÉSUMÉ La polychimiothérapie et les campagnes d'élimination de la lèpre sont des stratégies importantes pour vaincre la maladie. Nous présentons les résultats de la campagne d'élimination de la lèpre conduite en 2006 dans la province de Qazvin. Au total, 1987 personnes (1379 contacts issus du même foyer que les 319 patients enregistrés comme atteints de la lèpre et 608 personnes provenant de trois villages endémiques à forte prévalence) ont été examinées à des fins de dépistage de nouveaux cas de lèpre. Tous les nouveaux cas ont reçu une polychimiothérapie, puis ont été réexaminés après un an. Au cours de ce suivi, 256 cas suspects de lèpre ont été détectés, et 13 d'entre eux étaient des nouveaux cas confirmés (7 ont été classés comme des cas de lèpre multibacillaire). Aucun d'entre eux n'était porteur d'une déformation visible, ni âgé de moins de 20 ans. Tous les patients ont suivi le traitement de polychimiothérapie recommandé jusqu'à son terme. Le nombre réduit de cas détectés laisse à penser que dans les zones de faible prévalence, une approche sur le long terme comprenant des services de lutte antilépreuse intégrés et de prise en charge de l'incapacité serait une stratégie plus appropriée qu'une campagne d'élimination de la lèpre.

${ }^{7}$ Department of Infectious Diseases, Bou-Ali Hospital, Qazvin University ofMedical Sciences, Qazvin, Islamic Republic of Iran (Correspondence to R. Qasemi-Barqi: rqasemibarqi@yahoo.com).

${ }^{2}$ Department of Medical Microbiology, School ofMedicine, Qazvin University ofMedical Sciences, Qazvin, Islamic Republic of Iran.

Received: 14/10/09; accepted: 01/06/10 


\section{Introduction}

In 2004, data from 114 countries indicated that 407791 new cases of leprosy were detected, and globally there were altogether 286063 cases at the beginning of 2005 [1]. Approximately 2 to 3 million people are currently affected by active leprosy infection or post-infection sequelae worldwide [2].

Elimination of leprosy (reducing the prevalence of leprosy to less than 1 leprosy case per 10000 population) is the goal of the World Health Organization (WHO) [3]. By reducing the prevalence of leprosy to this level, it is expected that the transmission of Mycobacterium leprae will be interrupted leading to a gradual disappearance of the disease, although a number of authorities are less optimistic about such a hypothesis [4]. An alternative paradigm to controlling of leprosy that has been proposed is to live with leprosy while rendering it harmless [4].

The current approach to elimination of leprosy as a public health problem relies primarily on detection of patients with untreated hidden leprosy and treating sufferers with a multi-drug therapy (MDT) protocol. The WHO leprosy control programme is reported to have successfully accelerated the percentage of patients with leprosy who are receiving anti-leprosy drugs, which has resulted in a reduction in the prevalence of the disease worldwide [5].

In 1995, the WHO introduced a new initiative, Leprosy Elimination Campaign (LEC), which aims to detect and treat patients with hidden leprosy [6]. A review of LECs in 24 countries in 1999 showed that > 500000 new cases of leprosy were detected by LECs [6]. LECs also succeeded in promoting community awareness, reducing stigma and improving the accessibility of MDT and the skills of general health workers for diagnosis and treatment, especially in areas with poor care services [6]. After elimination of leprosy at the global level, the next challenge is to eliminate the disease at the national and district levels from every single country in the world [6].

The province of Qazvin is situated in north of the Islamic Republic of Iran in the southern part of the Alborz Mountains. It has a population of around 114 0000 (2006 census) living in 20 cities with 4 major cities comprising $18 \mathrm{sec}$ tions, 44 rural districts and 1543 villages. The national health services network in Qazvin province includes 289 rural health houses and 83 rural and urban health centres [7].

Leprosy control activities in the Islamic Republic of Iran began in 1958. This started in Qazvin through administration of dapsone monotherapy followed by the introduction of MDT in 1989 for treatment of leprosy. The prevalence of leprosy in the country in 2008 was calculated at 0.008 per 10000 population and Qazvin was one of the 3 provinces with a high detection rate [8].

The main purpose of this study was to identify whether the performance of a LEC had the potential to increase the detection of hidden cases in an endemic area compared to routine leprosy case detection activities.

\section{Methods}

This campaign was carried out between 1 March 2006 and 30 June 2006. The names and addresses of all patients with leprosy registered during 1958-2005 were obtained from the Qazvin Health Center (QHC) records (319 patients). The household contacts of the 319 cases were traced; there were 2871 contacts of whom 1379 were available. The 1379 household contacts together with 608 individuals from 3 endemic villages with a high prevalence for leprosy were examined by an expert team consisting of a general practitioner qualified in leprosy and a health technician qualified in both the clinical and laboratory aspects of leprosy with about 30 years of experiences.

All suspected cases were referred to the Infectious Diseases Ward, Bou-Ali General Hospital, Qazvin University of Medical Sciences and re-examined by a monitoring team (an infectious diseases specialist and a dermatologist). Suspicion of a case was based on observation of cutaneous manifestations (either with hypo- or hyperpigmented lesions). Cutaneous smears were taken from suspected individuals. Following acid fast staining, all smears were studied by an experienced microbiologist. Confirmed diagnosis was based on the Iranian Ministry of Health and Education guide, which was based on the WHO guide [9].

The cases with a confirmed diagnosis of leprosy were treated with MDT immediately. Duration of the treatment was according to WHO MDT guidelines [9].

Clinical examination of all the patients treated with MDT was carried out a year after LEC to assess the outcome of therapy.

\section{Results}

There were 258 suspected cases, of whom 13 were confirmed as new cases with 7 of the 13 classified as multibacillary (MB). None of the new cases was under 20 years of age or showed any visible deformities, all grade 0 (Table 1 ).

The reasons these 13 individuals were considered new cases of leprosy were: a) none of them had a history of anti-leprosy treatment (occurrence of relapse), and b) none of them had grade 2 disability (Table 1).

The incidence of leprosy in the Qazvin in 2005 was $0.03 / 10000$ population. During the LEC, 319 registered leprosy cases were found from 1958 to 2005. The incidence after the study at the end of the year 2006 was 1.1/10 000 population. 


\begin{tabular}{|c|c|}
\hline Variable & New cases (No.) \\
\hline \multicolumn{2}{|c|}{ Age (years) } \\
\hline 0-10 & 0 \\
\hline $11-20$ & 0 \\
\hline $21-30$ & 3 \\
\hline $31-40$ & 3 \\
\hline $41-50$ & 2 \\
\hline $51-60$ & 1 \\
\hline$>60$ & 4 \\
\hline \multicolumn{2}{|c|}{ Disability grade } \\
\hline 0 & 13 \\
\hline 1 & 0 \\
\hline 2 & 0 \\
\hline Total & 13 \\
\hline
\end{tabular}

All 13 patients confirmed with leprosy completed the recommended course of MDT (6 months for paucibacillary leprosy and 12 months for multibacillary leprosy according to the treatment guidelines of the National Leprosy Programme) with no need for further treatment [9].

The 13 patients after the completion of the anti-leprosy therapy, as with other leprosy patients, entered a support system including a state-run medical follow-up division and a social and economic advocacy facility, which gives support for leprosy patients through a charity foundation and also direct government financial aid.

\section{Discussion}

We found 13 new cases of leprosy in LEC, which is a considerable increase (4 in 2005 against 13 in 2006). Hence, this demonstrates the potential of LEC in detecting the hidden cases and indicates that LEC is a valuable strategic procedure for detecting hidden cases of leprosy in endemic area and also for household contacts. Despite

\begin{tabular}{lc}
\hline Table 2 Annual leprosy case detection in Qazvin province, 1996-2008 \\
\hline Year & New leprosy cases (No.) \\
1996 & 5 \\
1997 & 7 \\
1998 & 5 \\
1999 & 12 \\
2000 & 6 \\
2001 & 14 \\
2002 & 7 \\
2003 & 6 \\
2004 & 6 \\
2005 & 4 \\
2006 & 13 \\
2007 & 3 \\
2008 & 4 \\
\hline
\end{tabular}

the large increase in case detection, the prevalence of leprosy in Qazvin province is lower than the limit defined by WHO $(1 / 10$ 000) [3]. We failed to trace around $50 \%$ of the contacts of the leprosy cases and this represents a limitation of our study as and we may have missed other cases.

Some experts suggest that BCG vaccination plays a role in preventing leprosy $[10,11]$. All neonates in this region receive $B C G$ vaccination at the time of birth and because of the widespread distribution of primary health care (PHC) centres, the coverage of BCG vaccination has been $>99 \%$ since 1995. Therefore if BCG does play a role, this may explain the low number of cases in this area.

The main goal of the world leprosy programme is the elimination of leprosy at the global, national and district levels. Although some hypotheses indicate the possibility of infectious sources as well as patients [12], leprosy elimination is possible and the global strategy for this achievement is to detect and treat hidden cases of leprosy by MDT.MDT has been made available by WHO free of charge to all patients worldwide since 1995 and provides a simple yet highly effective cure for all types of leprosy [13]. Currently, MDT and LECs in high prevalence areas are considered the 2 major strategies for controlling and eliminating leprosy.

In a LEC programme in Nigeria during an 8-week case finding period in 1998, 323 new cases were detected, 24 of whom $(6.8 \%)$ were children, $236(67 \%)$ were multibacillary and 64 (18\%) had visible deformities [6]. Follow-up of patients placed on MDT in December 1999 revealed cure rates of 97\% for paucibacillary leprosy and 96\% for multibacillary leprosy. The authors concluded that LEC is cost-effective especially because it could be performed in a short period of time and utilized continuous media campaign to ensure that everyone learns about the signs of leprosy and its cure [6]. 
Similarly, in a modified LEC in the state of Orissa in India in 1998, 28.9 million people were examined and 62804 cases of leprosy were confirmed by experienced observers [14]. The total number of new cases detected and put on MDT treatment during only 7 days was approximately equal to that of a routine population survey by the leprosy services that would be recorded over a period of 2 years. In the 5 months following the completion of the campaign, the voluntary reporting rate increased from $50 \%$ to $90 \%$ [14].

In another LEC in 15 endemic areas of Amazonas state in Brazil in 1997, only 40 new cases were found. Compared to results in other states of Brazil, this number was low, possibly due to the development of health education activities and regular community services in the states of Amazonas [15].

In Table 2, the annual detected cases of leprosy found in the province of Qazvin (1996-2008) are shown. During this 13-year period, although 2 LECs were carried out throughout the province (2001 and 2006), there was no marked increase in the number of new leprosy cases found. Hence, it seems that in low prevalence areas such as Qazvin, LEC is not the most appropriate case-finding strategy.

All new cases detected in our LEC were over 20 years old. The absence of any patient $<20$ years in our study as well as the absence of any patients with grade 2 disability or higher (Tables $1 \& 3$ ) suggest that transmission of $M$. leprae in Qazvin is under control and those with current leprosy are people who were infected many years ago. This indicates that the leprosy elimination programme in this province has been successful over the past 2 decades (Tables 2 \& 3). A report by $\mathrm{WHO}$ indicated that at the end of 2005, 9.25\% of new cases in African regions and $8.2 \%$ in American regions were under 15 years of age $[16,17]$.

Although some authors believe that the disease is too complex to be eliminated by simple elimination programmes [18], we believe that the goal of elimination of leprosy is achievable and the key element is the integration of leprosy services into general health services in countries with a low prevalence. In the meantime, the performance of occasional LEC in endemic areas and among household contacts could increase the detection rate. Hence, the follow-up of household contacts and the residents in endemic areas through LEC activities by the health system is recommended.

In the post-elimination situation, WHO recommends a new strategy WHO Global Strategy 2006-2010 for Control of Leprosy. In addition to case detection and MDT, the new strategy emphasizes the prevention of leprosyrelated disabilities and rehabilitation through shifting from a campaignoriented elimination strategy toward a long-term approach of sustaining integrated quality leprosy services, disability management, reduction of stigma and discrimination through increased advocacy. The recently published global strategy has included new indicators for monitoring and evaluation of leprosy control activities, such as newly detected patients with grade 2 disability, treatment completion rate, and proportion of children among new cases [19].

\section{Conclusion}

LEC is considered to be of major importance in the elimination of leprosy, particularly in areas with a high prevalence of the disease. However, in low prevalence regions, it may not be the most appropriate and cost-effective approach. Instead, in such areas, efforts should be shifted from a campaignoriented strategy to long-term comprehensive and supportive (both medical and economic) services.

\section{Acknowledgements}

The authors would like to thank Dr Khorsand, Dr Kani, Dr Oskooei, Dr Leghaei, Mr Shahmoradi and, in particular, Mr Khoshkbarforoushha for their sincere cooperation in this study. 


\section{References}

1. Report of the seventh meeting of the WHO Technical Advisory Group on the Elimination of Leprosy (TAG). New Delhi, World Health Organization Regional Office for South-East Asia, 2005 (http://www.who.int/lep/resources/TAG7RPT.pdf, accessed 31 October 2011).

2. Kwan-Gett TSC, Kemp C, Kovarik C. Infectious and tropical diseases. St Louis, Elsevier Scientific, 2005:324.

3. World Health Organization Regional Office for South-East Asia. Leprosy frequently asked questions (http://www.searo. who.int/en/Section10/Section20/Section2000.htm, accessed 31 October 2011).

4. Rinaldi A. The global campaign to eliminate leprosy. PLoS Medicine, 2005, 2(12):e341.

5. Scollard DM et al. The continuing challenges of leprosy. Clinical and Microbiology Reviews, 2006, 19 (2):338-381.

6. Ebenso BE, Tureta SM, Udo So, Treatment outcome and impact of leprosy elimination campaign in Sokoto and Zamfara States, Nigeria. Leprosy Reviews, 2001, 72(2):192-198.

7. Iran 2006 Census (http://en.wikipedia.org/wiki/Qazvin_ Province, accessed 31 October 2011)

8. ILEP. Leprosy situation, IR Iran - 2008 (http://www.ilep.org uk/fileadmin/uploads/Country_Pages/Iran/Iran_01.pdf, accessed 31 October 2011).

9. A guide to eliminating leprosy as a public health problem, 2nd ed. Geneva World health Organization, 1997 [translated into Farsi by the Iranian Ministry of Health as a domestic guideline to eliminate leprosy.

10. Zodpey SP, Ambadekar NN, Thakar A. Effectiveness of Bacillus Calmette Guerin (BCG) vaccination in the prevention of leprosy: a population-based case-control study in Yavatmal District, India. Public Health, 2005, 119(3):209-216.

11. Cunha SS et al. Neonatal BCG protection against leprosy: a study in Manaus, Brazilian Amazon. Leprosy Reviews, 2004, 75(4):357-366.

12. Lockwood DN. Leprosy elimination - a virtual phenomenon or a reality? British Medical Journal, 2002, 324(7352):1516-1518.

13. Novartis Foundation For Sustainable Development. Q\&A about the Novartis MDT leprosy donation (http://www. novartisfoundation.org/page/content/index.asp?MenulD= $364 \& I D=1010 \&$ Menu $=3 \&$ Item $=43.2 .4$, accessed 31 October 2011).

14. Patnik PKB. Modified Leprosy Elimination Campaign (MLEC) in the state of Orissa, India. Leprosy Reviews, 1999, 70(4):440443.

15. Dos $\mathrm{S}$ et al. Leprosy elimination campaign, Amazonas-Brazil 1997. Leprosy Reviews, 2000, 71(1):77-80.

16. African region: leprosy situation at the end of 2006 (www. who.int/lep/situation/AFROStatsEnd2006.pdf, accessed 31 October 2011).

17. American region: leprosy situation at the end of 2006. www. who.int/entity/lep/situation/AMROStatsEnd2006.pdf, accessed 31 October 2011)

18. Lockwood DNJ, Suneetha S. Leprosy too complex a disease for a simple elimination paradigm. Bulletin of the World Health Organization, 2005, 83:230-235.

19. Global strategy for further reducing the leprosy burden and sustaining leprosy control activities 2006-2010. Geneva, World Health Organization, 2005. 